\title{
Ultrafast dynamics of carrier-induced absorption changes in highly-excited CdSe nanocrystals
}

\author{
S. Hunsche ${ }^{\star}$, T. Dekorsy, V. Klimov, H. Kurz \\ Institut für Halbleitertechnik II, Rheinisch-Westfälische Technische Hochschule Aachen, D-52056 Aachen, Germany \\ (Fax: +49-241/8888246, E-mail: HUNSCHE@basl.rwth-aachen.de)
}

\begin{abstract}
We use femtosecond time-resolved transmission spectroscopy to study the density and time dependence of transient absorption changes of CdSe nanocrystals. Our data show pronounced absorption saturation up to complete bleaching of the lowest optical transition. At high carrier density the nonlinear spectra show several peaks that can be related to the two lowest quantized electron states. Thetime dependence of the carrier-induced absorption changes indicates an ultrafast relaxation process within the strongly broadened absorption lines.
\end{abstract}

PACS: 78.47.+p; 73.20.Dx; 71.35.+z

In recent years, there has been a strong interest in lowdimensional semiconductor structures such as quantum wells, quantum wires, and quantum dots. The electronic states in these artificial materials are strongly influenced by size quantization effects, which occur when the dimensions of the structures become comparable to or smaller than the exciton Bohr radius $\mathrm{a}_{\mathrm{B}}$. Then, the electronic and optical properties of the structures become determined by their geometry. Thus, the controlled fabrication of low-dimensional semiconductor structures may allow to obtain materials with predefined properties.

In addition, size quantization has been shown to lead to a significant enhancement of excitonic effects and to strongly enhanced optical nonlinearities in two-dimensional quantum wells, where the carriers are confined in only one dimension [1]. It can be expected that a confinement of carriers in two or three dimensions will lead to a further enhancement of the optical nonlinearities. Therefore, semiconductor quantum dots appear as a promising new material for nonlinear optical applications. It has been found that semiconductor crystallites with radii on the order of a few nanometer, denoted as nanocrystals (NCs), can be rather easily produced in semiconductor-doped glasses (which are commercially available as color-glass filters) or in organic solution [2-4].

* Present address: FOM-Institute for Atomic and Molecular Physics, Kruislaan 407, 1098 SJ Amsterdam, The Netherlands
The linear absorption of such samples generally shows a correlation with the average NC size, as the lowest absorption feature shifts towards higher energies with decreasing size $[3,5-11]$. While this clear sign of size quantization is easily observed, the absorption spectra of semiconductordoped glasses usually fail to show sharp exciton peaks (in contrast with two-dimensional quantum wells) or even wellseparated absorption lines that reflect the expected discrete energy spectrum of a "zero-dimensional" structure. It is commonly accepted that the presence of discrete electronic states is masked in the linear absorption by strong homogeneous and inhomogeneous broadening. The nanocrystals may vary in size, geometry, and chemical composition. These variations cause a strong inhomogeneous broadening of the optical transitions. Variations of the NC size are typically on the order of $25 \%$ [12]. This value may be reduced to a few percent by sophisticated preparation techniques, but even then the size distribution has a significant influence on the optical properties $[4,10,13]$. In addition, electron-phonon coupling and the large number of "dangling bonds" at the surface of the NCs may lead to a considerable homogeneous broadening that is at least comparable to the inhomogeneous broadening at room temperature [5, 14].

A large amount of experimental and theoretical work has been done to determine the absorption and luminescence properties of semiconductor NCs and to gain insight in their energy structure and dominant broadening mechanisms [3, $7-11,13]$. The magnitude and the dynamics of the optical nonlinearities have been investigated with various experimental techniques, such as single-beam absorption saturation, [15] pump-probe measurements in the nanosecond, $[4,6,7,9,10,16]$ picosecond, $[5,17,18]$ and femtosecond regime, $[4,19]$ and photon-echo experiments $[11,14,20]$.

It has been found that the optical nonlinearities may depend strongly on experimental details such as temperature, surface properties, details of the fabrication process, and even illumination history. This latter effect is observed as "photodarkening" by strong laser illumination which leads to a weak broadband reduction of the linear transmission $[15,21]$ and to drastically reduced lifetimes of optically excited carriers, resulting in a reduction of the luminescence 
intensity and of the carrier-induced absorption changes in the nanosecond time regime [16, 21-23].

Also, a large amount of theoretical work has been done using various refinements of the first effective-mass calculations $[3,24,25]$ in order to understand the optical properties of semiconductor NCs $[26-30,8,31]$. In spite of these efforts, the agreement between theory and experiments generally remains rather qualitative, indicating that the insight in the physics of semiconductor NCs is still incomplete. New, detailed experimental data may help to improve the understanding of these promising materials and to gain a better insight in their electronic structure and the interactions that govern the carrier dynamics in semiconductor NCs.

In this paper, we present nonlinear transmission measurements of CdSe nanocrystals after optical excitation with high-intensity femtosecond laser pulses. We investigate the excitation density dependence and the dynamics of carrierinduced absorption changes after initial photodarkening of the sample. We observe a strong absorption saturation, up to complete bleaching of the lowest optical transition, and find that the bleaching is dominated by the occupation of electron states at room temperature. While the structure of the nonlinear absorption spectra reflects a basically discrete spectrum of electron states, the time dependence surprisingly indicates a relaxation mechanism within the corresponding absorption lines. This may possibly be explained by a strong coupling of the discrete levels to a quasi-continuum of vibrational or surface states. The manuscript is organized as follows: In Sect. 1, we briefly describe the experimental details. The experimental results are presented in Sect. 2 and discussed in Sect. 3. Our conclusions are summarized in Sect. 4.

\section{Experimental setup}

The pump-probe experiments are performed using an amplified $(5 \mu \mathrm{J} /$ pulse; $6.8 \mathrm{kHz}$ repetition rate) Colliding-Pulse Mode-locked (CPM) laser to excite the sample with femtosecond pulses of $60 \mathrm{fs}$ duration and a photon energy of $2 \mathrm{eV}$. Pump-induced transmission changes are measured by time-delayed "white-light" continuum pulses which are created by focusing a part of the amplified pulses into an ethylene glycol jet. We use an Optical Multichannel Analyzer (OMA) system to record the differential transmission spectra: DTS $=\Delta T(\tau) / T_{0}$, at a fixed time delay $\tau$ between pump and probe pulses. Here, $T_{0}$ and $T$ denote the transmission spectrum with and without excitation, respectively. The transmission changes are directly related to changes of the optical density via: DTS $\approx \mathrm{e}^{-\Delta \alpha d}-1$. All DTS are measured after some minutes of strong illumination of the probe spot with a pump fluence of several $\mathrm{mJ} / \mathrm{cm}^{2}$ per pulse, so that the sample is completely photo-darkened (Sect. 2.2). We measure the DTS seperately for probe energies above and below the pump energy because of strongly different probe intensities in these energy ranges. Around time delay zero, the DTS are slightly disturbed due to the chirp of the probe continuum, which reduces the overall time resolution to $\approx 200 \mathrm{fs}$. We have made additional pump-probe measurements using unamplified CPM pulses at $2 \mathrm{eV}$ and a fast-scan measurement scheme, where a time resolution of $50 \mathrm{fs}$ and a signal-to-noise ratio of $10^{-5}$ are obtained with an integration time of less than one minute. Details of this technique

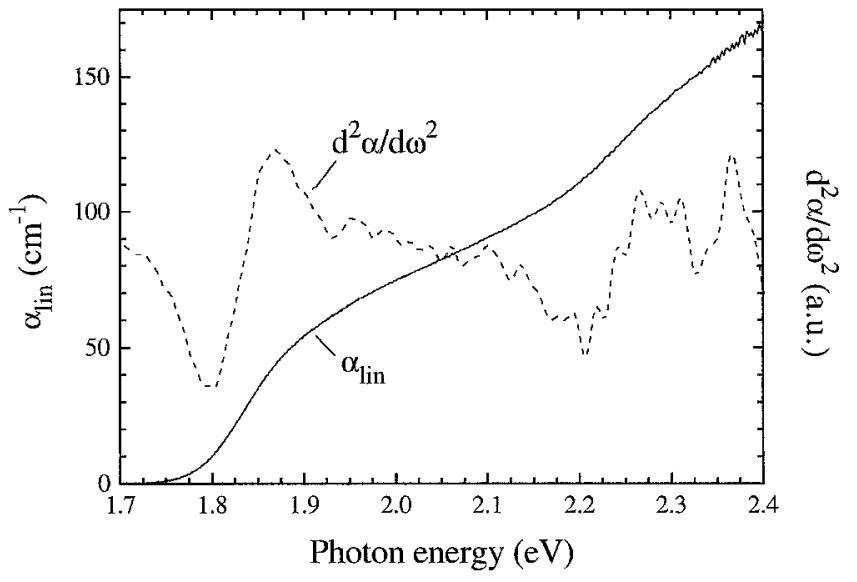

Fig. 1. Linear absorption and second derivative of the absorption of the CdSe NC sample

are given in [32]. All measurements are performed at room temperature.

The sample is a $280 \mu \mathrm{m}$ thick platelet of commercially available filter glass (KC-19), which is nominally doped with $\mathrm{CdS}_{x} \mathrm{Se}_{1-x}$. Transmission electron microscopy shows that the sample contains semiconductor NCs with an average radius of $5.7 \mathrm{~nm}$. The standard deviation from this average radius was estimated to be $\approx 10 \%$. The NC radius is on the order of the bulk CdSe exciton Bohr radius, which is $5.6 \mathrm{~nm}$. Since Raman spectra of the sample show only CdSe lattice vibrations, we assume the sample to contain pure CdSe NCs. The linear absorption of the sample is $80 \mathrm{~cm}^{-1}$ at $2 \mathrm{eV}$, i.e., $0.13 \%$ of the bulk CdSe absorption. Taking into account a local field correction $f$, the volume fraction of CdSe in the glass matrix can be estimated to be $p=\alpha_{\mathrm{NC}} / \alpha_{\text {bulk }}|f|^{2}$ [29]. With $|f|^{2} \approx 0.4$, for spherical CdSe NCs, this yields a concentration of $\approx 0.3 \%$ in our sample.

\section{Experimental results}

\subsection{Linear absorption}

The linear absorption spectrum of the sample has been measured using a commercial spectrometer and is shown in Fig. 1. We also show the second derivative of the absorption, $d^{2} \alpha / d \omega^{2}$. Obviously, the sample exhibits a blue-shift of the absorption edge with respect to the bulk CdSe band gap of $1.751 \mathrm{eV}$ at room temperature, indicating a quantum confinement of carriers in the NC's. However, the absorption spectrum resembles that of a bulk semiconductor and shows no sharp structures. The second derivative spectrum shows two weak maxima at 1.86 and $2.27 \mathrm{eV}$. The energy difference of these structures agrees with the energy splitting between the $\mathrm{A}$ (heavy hole) and $\mathrm{C}$ (split-off) valence bands in bulk $\mathrm{CdSe}$, suggesting that these structures are related to the lowest optical transitions from the $\mathrm{A}$ and $\mathrm{C}$ valence bands, respectively, to the conduction band. There is no clear indication of other discrete, separated transitions in the linear absorption. 


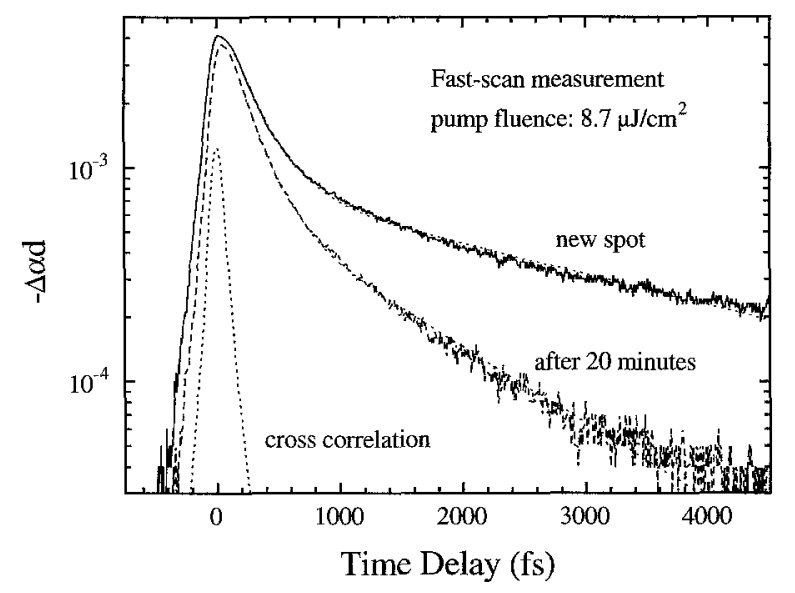

Fig. 2. Dynamics of absorption changes measured with unamplified CPM pulses at $2 \mathrm{eV}$

\subsection{Photodarkening}

In Fig. 2, we show transient absorption changes, measured using unamplified CPM pulses for excitation and probing in a fast-scan setup. The pump was focused to a spot of 30 $\mu m$ diameter, resulting in a pump fluence of $8.7 \mu \mathrm{J} / \mathrm{cm}^{2}$. Trace $\mathrm{A}$ has been measured on a previously unexposed spot of the sample and trace B has been obtained on the same spot after $20 \mathrm{~min}$ of illumination with pump and probe beams. The duration of each measurement was less than one minute. The pump-probe cross correlation can be well fitted assuming sech $^{2}$-pulses of $45 \mathrm{fs}$ duration. Both absorption transients show a bleaching at the pump wavelength, which relaxes with an initial fast and a second, much larger, time constant. The main difference between traces $\mathrm{A}$ and $B$ is a drastic reduction of the slow decay rate, while the maximum amplitude and the initial fast absorption recovery time do not change much. Fitting the decay of the bleaching with a double exponential gives initial time constants of $240 \mathrm{fs}$ for curve A and $200 \mathrm{fs}$ for curve B, and slow decay time constants of $3 \mathrm{ps}$ for curve $\mathrm{A}$ and $1.2 \mathrm{ps}$ for curve $B$. Further illumination of the sample did not change the time dependence observed in curve B. Also, the measured absorption dynamics showed only a minor recovery after 1 h without laser exposure. Obviously, the dynamics of the nonlinearities of our sample are modified by laser-induced "darkening", which results in the opening of a non-radiative recombination channel. The darkening effect occurs much faster under illumination with higher probe fluences. In our DTS measurements, which have been performed with much larger excitation densities than the fast-scan experiment, we observed a reduction of the bleaching amplitude (at positive delay time) within less than a minute. In an experiment using frequency-doubled pump pulses [33], the visible luminescence decayed within a few seconds. All nonlinear spectra shown in the following are obtained after strong illumination of the probe spot for a few minutes, so that the sample is completely darkened.

\subsection{Density dependence}

In contrast to the linear absorption spectrum, the nonlinear absorption clearly shows discrete structures, as illustrated

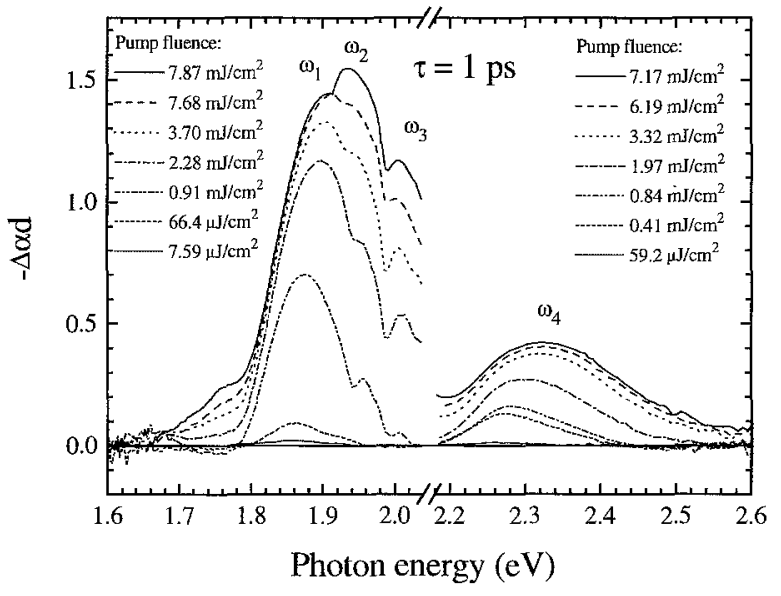

Fig. 3. Absorption changes of the NC sample at various pump fluences and at a fixed time delay of $1 \mathrm{ps}$ between pump and probe

in Fig. 3. There, we present carrier-induced changes of the optical density, $-\Delta \alpha d$, derived from DTS taken at various pump intensities between $7.59 \mu \mathrm{J} / \mathrm{cm}^{2}$ and $7.87 \mathrm{~mJ} / \mathrm{cm}^{2}$. The pump intensities have not been exactly the same in the measurements of the high- and low-energy spectra (above and below $2.1 \mathrm{eV}$, respectively), as indicated in the figure. All spectra are taken at a fixed time delay of $1 \mathrm{ps}$. From our time-dependent DTS measurements (Sect. 2.4), we conclude that carrier thermalization and cooling occur on a much shorter time scale. Hence, the spectra in Fig. 3 essentially show the dependence of the absorption changes on carrier density. The spectra show strong absorption saturation in the energy ranges from 1.8 to $2.1 \mathrm{eV}$, and from 2.2 to $2.5 \mathrm{eV}$, i.e., around the energies of the structures observed in the second derivative absorption spectrum. At high pump intensities, the low-energy bleaching shows clearly resolved peaks. In the following, we will denote the bleaching bands by $\omega_{1}$ to $\omega_{4}$, corresponding to the peaks at $1.86,1.94,2.01$, and $2.3 \mathrm{eV}$. The energetically lowest of these structures has a nearly Gaussian shape at high pump intensities, with the $\omega_{2}$ and $\omega_{3}$ peaks superimposed on its high energy edge. At low excitation density, the two latter structures disappear and the $\omega_{1}$ band becomes more asymmetric. The asymmetry of the bleaching band at low intensities suggests a further weak transition at $1.9 \mathrm{eV}$ which overlaps with the lowest peak resulting in a single bleaching band at higher excitation densities. In contrast, the absorption saturation above $2.2 \mathrm{eV}$ appears as a single asymmetric bleaching band at all pump intensities. At low pump intensities, we observe a weak induced absorption between the two bleaching bands, as in previous experiments $[4,19]$. However, the nonlinear absorption in our experiments is obviously dominated by carrier-induced bleaching rather than possible broadening or energy shift of the optical transitions.

A striking fact visible in these spectra is the clear shift of both the low- and the high-energy bleaching maximum with varying pump intensity. The low-energy bleaching peak position continuously shifts from 1.86 to $1.89 \mathrm{eV}$, and the high-energy maximum from 2.26 to $2.33 \mathrm{eV}$ with increasing intensity. These intensity-dependent spectral changes of the nonlinear absorption neither match the characteristic "spectral hole burning" within an inhomogeneous absorption band 


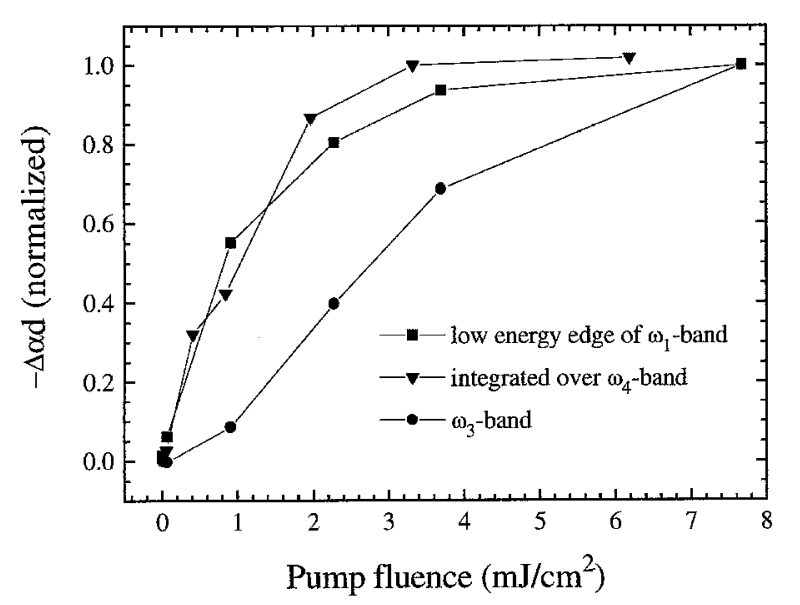

Fig. 4. Density dependence of pump-induced absorption changes at different probe energies

nor the uniform saturation of a single homogeneously broadened transition. The observed density dependence rather suggests a gradual band-filling, due to an increasing thermal occupation of a quasi-continuum of electronic states. The $\omega_{1}$ bleaching band saturates at the largest pump intensities, while the $\omega_{2}$ and $\omega_{3}$ peaks still increase with intensity above $\approx 7 \mathrm{~mJ} / \mathrm{cm}^{2}$. The maximum absorption change at the lowenergy edge of the $\omega_{1}$ band matches almost exactly the linear absorption, i.e., $\Delta \alpha \simeq \alpha_{\text {lin }}$ in the range 1.8 to $1.9 \mathrm{eV}$. The spectra taken at pump intensities above $3.7 \mathrm{~mJ} / \mathrm{cm}^{2}$ show a complete bleaching in this energy range, but no significant optical gain. The bleaching band around $2.3 \mathrm{eV}$ essentially shows the same behaviour as the $\omega_{1}$ band. Besides the shift of the bleaching maxima, we find the same density dependence of these two bleaching bands. This is illustrated in Fig. 4, which shows the density dependence of the bleaching amplitudes at the low-energy edge of the $\omega_{1}$ band and of the $\omega_{3}$ and $\omega_{4}$ bands. The data for the $\omega_{4}$ band are corrected for the broadband bleaching background present at high pump intensities (Fig. 3). Both the $\omega_{1}$ and the $\omega_{4}$ bands are characterized by a saturation of the bleaching amplitude at a pump fluence of $3-4 \mathrm{~mJ} / \mathrm{cm}^{2}$. The density dependence of the $\omega_{3}$ bleaching is strongly different, as already concluded from Fig. 3. This bleaching band becomes significant only at higher densities and does not saturate in the intensity range covered by our experiments. According to the spectra, the density dependence of the $\omega_{2}$ band is very similar to that of the $\omega_{3}$ band. However, the bleaching amplitude is strongly influenced by the overlap with the high energy edge of the $\omega_{1}$ band. Therefore, the normalized $\omega_{2}$ data lie between those of the of the $\omega_{3}$ band and the $\omega_{1}$ band, and are not shown in Fig. 4.

\subsection{Time dependence}

In Fig. 5, we show the time dependence of the pump-induced absorption changes after excitation with a pump fluence of $0.32 \mathrm{~mJ} / \mathrm{cm}^{2}$ per pulse. The DTS experiments did not show any clear sign of carrier relaxation from the photogeneration energy to lower-energy states. This indicates that carrier thermalization and cooling occur within the first $\approx 200 \mathrm{fs}$, in agreement with the fast time constant observed in the fast-

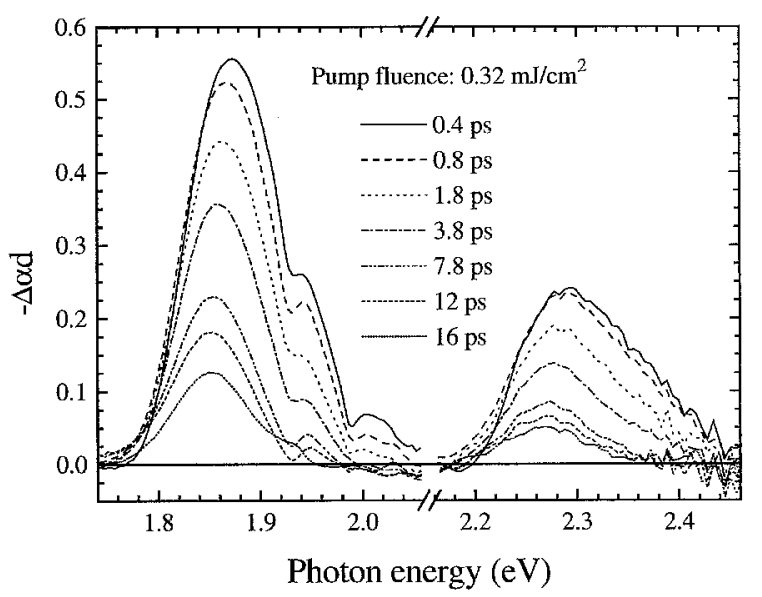

Fig. 5. Pump-induced absorption changes at various time delays $\tau$ between pump and probe. The pump fluence is $0.32 \mathrm{~mJ} / \mathrm{cm}^{2}$ per pulse

scan experiments (Fig. 2). Therefore, we interpret this fast time constant as the energy relaxation of carriers out of the photo-excited into lower-energy states, i.e., carrier thermalization and cooling.

The time-dependent DTS show the same bleaching bands as the density-dependent measurements after the pump pulse has passed. With increasing time delay, we observe a fast reduction of the bleaching amplitude which occurs on a time scale of a few picoseconds. The spectra at longer time delays closely resemble spectra taken at small delay, but with lower pump intensity. Therefore, we conclude that all DTS correspond to thermal distributions of the pump-excited carriers and that the time dependence of the bleaching mainly reflects the time evolution of the carrier density. The dynamics of the absorption changes are determined by a very fast decay of the photoexcited carrier density. Like the spectra taken at different densities, the time-dependent spectra show an energy shift of the bleaching peaks; with increasing time delay the $\omega_{1}$ and $\omega_{4}$ maxima shift towards the low-energy edge of the bleaching bands observed at high density. This observation suggests that the dynamics is determined by carrier recombination plus an additional energy relaxation within the bleaching bands.

The dynamic red-shift corresponds to a faster absorption recovery at higher probe energies, as illustrated in Fig. 6. Here, we present the dynamics of the carrier-induced absorption changes at different probe energies within the $\omega_{1}$ and $\omega_{4}$ band. Taking the energies of the bleaching maxima at long time delay $(1.85$ and $2.27 \mathrm{eV})$ as a reference, the transients shown in Figs. 6a and b correspond to equivalent positions within the two bleaching bands. In both bands, we find a continuous variation of the absorption recovery times, which become much shorter with increasing probe energy. The bleaching decay at $2.0 \mathrm{eV}$ (not shown in Fig. 6) agrees with the $1.2 \mathrm{ps}$ time constant observed in the fast-scan experiment. The transients in Figs. $6 \mathrm{a}$ and $\mathrm{b}$ are practically identical for equivalent positions in the respective band; consequently, we also observe the same dynamics of the total absorption changes that are obtained by integrating the $\Delta \alpha$ spectra over the width of the $\omega_{1}$ and $\omega_{4}$ bands. These integrated absorption changes can be taken as a measure of the occupation of the corresponding carrier states, and are 

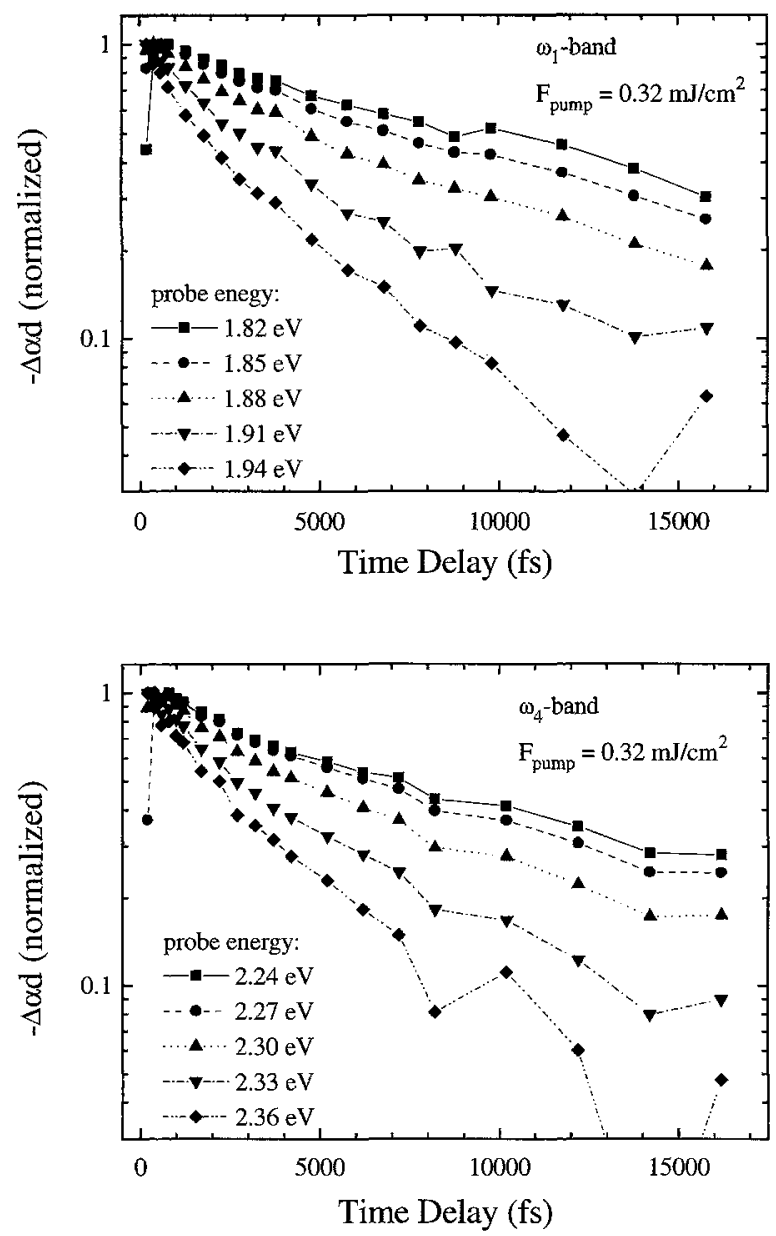

Fig. 6a,b. Time dependence of pump-induced absorption changes at different probe energies. (a) At different positions within the low-energy bleaching band $\left(\omega_{1}\right)$; (b) within the high-energy bleaching band $\left(\omega_{4}\right)$

shown as squares and circles, repectively, in Fig. 7. For a better comparison of the temporal evolution, these data are normalized to the maximum change at $\Delta t=0$. The normalization factor for each curve is given in the plot. Clearly, the $\omega_{1}$ and $\omega_{4}$ bands have not only the same density dependence, but also show a practically identical time dependence, indicating that the occupation of one common electronic level determines the bleaching of both bands. The slight deviation at long delay times may be caused by an additional weak induced absorption above the lowest bleaching structure that has been observed in many previous experiments.

The dynamics of the absorption recovery cannot be described by a single exponential decay. The decay of the transients measured with a pump intensity of $0.32 \mathrm{~mJ} / \mathrm{cm}^{2}$ clearly becomes slower at longer delay times. This behavior might suggest a strongly density-dependent recombination mechanism, such as Auger recombination [34-36]. However, plotting $(1 / \Delta \alpha)^{2}$ as a function of time delay [36] does not give a straight line, indicating that the decay is not dominated by an Auger term. The normalized data can be fitted equally well by a double-exponential (dashed lines in Fig. 7) or a mixed exponential-Auger decay (dotted line) described by [36]:

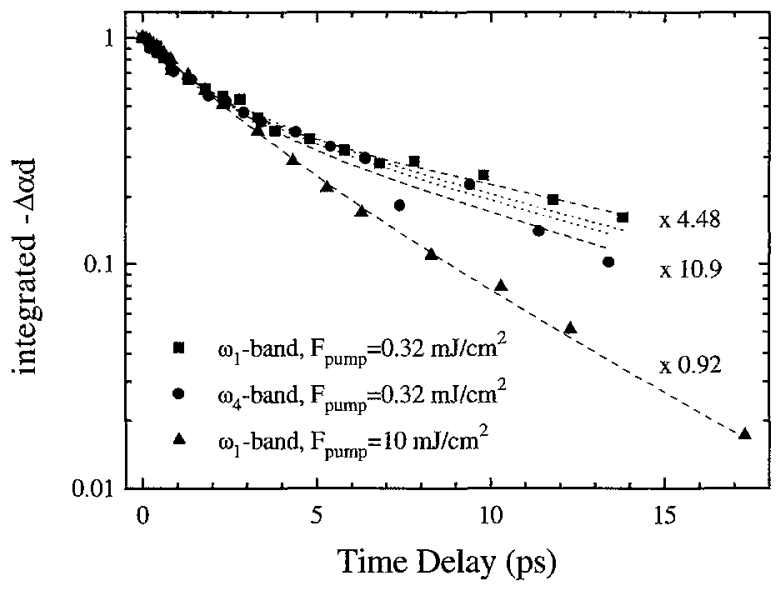

Fig. 7. Dynamics of integrated absorption changes obtained from DTS at different excitation densities. Dashed and dotted curves are numerical fits of the experimental data (see text)

$\frac{1}{N^{2}}=\mathrm{e}^{2 t / \tau}+C \tau\left(\mathrm{e}^{2 t / \tau}-1\right)$

assuming that $N(t=0)=1$. The latter fitting procedure gives almost identical values of $\tau \approx 11 \mathrm{ps}$ and $C=0.35 \mathrm{ps}^{-1}$ for both bleaching bands, while the double exponential fit leads to time constants of $\tau_{1}=1.6 \mathrm{ps} ; \tau_{2}=12 \mathrm{ps}$ and $\tau_{1}=1.8 \mathrm{ps}$; $\tau_{2}=9.2 \mathrm{ps}$ for the $\omega_{1}$ and $\omega_{4}$ band, respectively. Taking into account the fluctuations of the $\omega_{4}$ data, the limited delay range and the possible influence of a weak induced absorption, the differences are not really significant.

In order to check the possible importance of Auger recombination, we compare the low-density data with data obtained with a much higher excitation density. The triangles in Fig. 7 show the transient absorption changes integrated over the $\omega_{1}$ band, after excitation with a pump fluence of $10 \mathrm{~mJ} / \mathrm{cm}^{2}$. While the maximum bleaching (of the nonnormalized data) is about five times higher than the value measured at low density, the initial decay dynamics remains almost unchanged. This demonstrates that the absorption recovery in our experiments is clearly not influenced by Auger recombination, which should lead to an 25 -fold reduction of the initial decay time constant. A double-exponential fit gives $\tau_{1}=2.3 \mathrm{ps}$ and $\tau_{2}=5.1 \mathrm{ps}$, while the data cannot be fitted well using (1).

Surprisingly, at long delay times, the decay in the high-density measurement remains quite fast, even after the bleaching amplitude has dropped below the values obtained in the low-density experiment. This leads to a crossing of the curves (in the non-normalized data) at $\approx 13$ ps which can clearly not be explained by the higher carrier density. However, previous picosecond pump-probe measurements with the same sample showed an extreme temperature dependence of the carrier lifetimes. A value of 2 ns has been found at $77 \mathrm{~K}$, which is reduced to only $40 \mathrm{ps}$ at room temperature, indicating the thermal activation of a fast non-radiative recombination process $[17,18]$. Therefore, we believe that sample heating may be the main reason for the average reduction of the decay time constants under high-intensity excitation. A rough estimation of the pump-induced temperature increase, on the basis of the average absorbed intensity and the thermal conductivity of glass, gives a value on the order of $10 \mathrm{~K}$. 
While this may be sufficient to cause the observed change of the decay dynamics, the corresponding shift of the band edge can not be detected in the DTS because of the large width of the bleaching bands.

\section{Discussion}

The first striking observation in the DTS is the occurrence of several discrete peaks, in contrast to an essentially unstructured linear absorption spectrum. Similar findings have previously been interpreted in terms of spectral hole burning within an inhomogeneously broadened absorption line, which corresponds to a selective excitation of a certain fraction of the NC size distribution.

However, this model can be excluded as an explanation of our experiments by a number of arguments. First of all, we do not observe spectral hole burning at the pump energy which is clearly above the lowest optical transition. The pump-induced bleaching occurs at the low-energy edge of the absorption spectrum, i.e., at the low-energy edge of the lowest optical transition. Explaining this result in the selective excitation picture would require that the pump energy coincides (only) with the low-energy edge of some specific higher transition. There is no experimental indication that this is the case. Secondly, the bandwidth of the femtosecond pump pulses, which have a full width at half maximum of $\approx 45 \mathrm{meV}$, is larger than the inhomogeneous broadening for a typical size variation of $25 \%$, which can be estimated to be $30-35 \mathrm{meV}$ for an average radius of $6 \mathrm{~nm}$ [12]. In addition, higher optical transitions are expected to be rather closely spaced, so that an overlap of the pump with a part of one isolated inhomogeneously broadened absorption line seems quite unlikely. Furthermore, the observed complete bleaching of the lowest transition under high-intensity pumping could only be interpreted as a coincidence when excitation of a limited number of NCs is assumed. Finally, the DTS of our sample exhibit the same structures after excitation with a pump photon energy of $4 \mathrm{eV}$ as under $2 \mathrm{eV}$ excitation [33].

However, the density dependence of the spectra clearly shows that the absorption changes are due to carrier-induced bleaching of the lowest optical transitions. An optically induced build-up of an electric field within the NCs, as proposed in [10] can be ruled out since this field would be screened, not enhanced, by an increase of the excitation density that leads to the excitation of further $\mathrm{c}-\mathrm{h}$ pairs. Therefore, the DTS contain information about the lowest energy levels only. In contrast, the linear absorption is a superposition of a larger number of different transitions that can be expected to be rather closely spaced and strongly broadened at higher energies, leading to a practically continuous linear absorption spectrum.

In contrast, the discrete structures observed in the DTS reflect the underlying discrete energy structure of the NCs and allow to obtain information about specific states. Since the $\omega_{1}$ and $\omega_{4}$ bands show essentially the same time and density dependence, these bleaching structures must be associated with the same, lowest, electronic level, while the $\omega_{2}$ and $\omega_{3}$ bands can be assigned to a higher state that is only occupied after high-energy [33] or high-density excitation. In principle, a straightforward assignment of the observed structures is possible on the basis of the simple strong-confinement approximation, in which electrons and holes are described by identical wavefunctions [3, 24]. Different quantization energies arise from the different effective masses and dipole-allowed optical transitions occur only between levels with the same principal and momentum quantum numbers.

Within this model, the occupation of the lowest $(1 s)$ electron state should lead to a bleaching of three optical transitions associated with the three valence bands of $\mathrm{CdSe}[6,19]$. Considering literature values for the various effective masses [37], and a $\mathrm{NC}$ radius of $6 \mathrm{~nm}$, the strong confinement model predicts the following transition energies for the lowest optical transitions: $1 s-1 s_{\mathrm{A}}, 1.83 \mathrm{eV} ; 1 s-1 s_{\mathrm{B}}, 1.88 \mathrm{eV}, 1 p-1 p_{\mathrm{A}}$, $1.94 \mathrm{eV}, 1 p-1 p_{\mathrm{B}}, 2.02 \mathrm{eV}, 1 s-1 s_{\mathrm{C}}, 2.27 \mathrm{eV}$. These values agree quite well with the positions of the bleaching bands in our DTS data, assuming that the $1 s_{\mathrm{A}^{-}}$and $1 s_{\mathrm{B}}$-transitions merge into a single bleaching band.

Recent, more detailed theoretical studies indicate that the strong-confinement model is too simple and that details of the valence-band structure have to be taken into account. These calculations predict considerable changes of the selection rules and a larger number of transitions related to the lowest electron state [8]. These predictions have been supported by experimental data in [10], where five bleaching peaks coupled to the $1 s$ electron state are reported. However, the pump-probe data presented there show mainly a broadening of all optical transitions and are interpreted in terms of build-up of an electric field within the NCs. Quite surprisingly, the data do not show several transitions associated with the $1 p$ electron state. While the energies of the four bleaching bands observed in our DTS agree also quite well with the transition energies reported in [10] (also assuming that the lowest two transitions merge into a single band), the density dependence clearly shows that the $\omega_{2}$ and $\omega_{3}$ bands cannot be assigned to the $1 s$ electron state.

However, the results of [8] indicate that the oscillator strength of the $1 S_{1 / 2}-1 S_{\mathrm{e}}$ and $2 S_{1 / 2}-1 S_{\mathrm{e}}$ transitions (in the notation of [8]) vanish and that the $3 S_{1 / 2}$ hole level approaches the maximum of the $\mathrm{C}$-valence band for large quantum dots. Obviously, the influence of valence-band mixing decreases with NC size so that the detailed calculation and the strong-confinement model appear to become equivalent for large NCs. In fact, also the positions of the $\omega_{2}$ and $\omega_{3}$ bands in our DTS agree quite well with the energies of the two lowest $1 P_{\mathrm{e}}$ transitions predicted by both models. Therefore, our data do not allow a discrimination between the models and the only differences are in the different labeling of the transitions. In the following, we keep the more intuitive picture of three valence bands as in the strongconfinement model [38].

Clearly, the $\omega_{4}$ bleaching band can only be caused by pump-excited electrons since the transition energy is higher than the excitation energy and no holes are excited into Cband states. From the time and density dependences which are essentially identical for the $\omega_{1}$ and $\omega_{4}$ band, it can be concluded that the bleaching is dominated by electrons rather than holes for both bands. Considering that the bleaching is related to the sum of electron and hole occupation numbers, $f_{\mathrm{e}}+f_{\mathrm{h}}$, this means that $f_{\mathrm{h}}$ is much smaller than $f_{\mathrm{e}}$. This interpretation is consistent with the observation that 


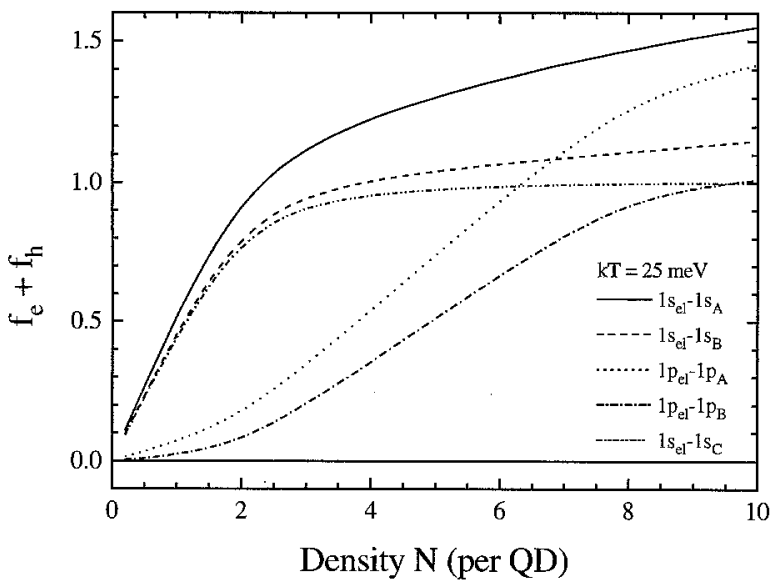

Fig. 8. Density dependence of the occupation factor $f_{\mathrm{e}}+f_{\mathrm{h}}$ for various transitions, calculated within the strong-confinement approximation

the maximum absorption changes of the $\omega_{1}$ band under highdensity excitation coincide with the linear absorption. The non-observation of optical gain indicates that $f_{\mathrm{e}}+f_{\mathrm{h}} \approx 1$, which might be taken as an indication of hole trapping, as proposed previously [4].

However, a much smaller hole occupation can also arise from the much higher (average) density of states in the valence bands, similar as in a bulk semiconductor [39]: Due to the larger valence-band effective masses, the hole levels are much narrower spaced than the electron levels. At sufficiently high temperature, photoexcited holes will be thermally distributed over a large number of levels, resulting in much smaller hole occupation numbers. This picture is supported by picosecond pump-probe measurements, where optical gain has been reported at low temperature, but could not be observed at room temperature [17, 18].

In order to understand the density dependence of our experimental data, we have calculated the filling factors $f_{\mathrm{e}}+f_{\mathrm{h}}$ for a spherical quantum dot. For simplicity, we used the strong-confinement model to calculate the electron- and hole-energy spectrum for a $6 \mathrm{~nm} \mathrm{NC}$ and assumed a thermal occupation of the electron and hole states at room temperature. Figure 8 shows the filling factors of the dipole-allowed transitions that correspond to the bleaching bands observed in the DTS. Obviously, the simple model gives a good qualitative explanation for the measured density dependence of the bleaching bands, as shown in Fig. 4. In particular, the occupation factor for the lowest transition rises almost linearly up to a value close above 1 , and then shows only a very slow increase with carrier density above $2-3$ electron-hole pairs per NC. In contrast, the bleaching of the $1 p$-related transitions becomes significant only after the near-saturation of the $1 s$ electron level, and saturates at much higher carrier density. Clearly, the occupation factor is dominated by occupation of electron states, since the holes are distributed over a larger number of levels at room temperature. We note that this is no specific prediction of the strong-confinement model, since including corrections due to, e.g., Coulomb interaction or details of the valence bands will not significantly change the fact that the mean hole density of states (averaged over a finite energy interval) is much larger than that of the electrons.
Considering that the general structure of the DTS can be quite well understood in terms of discrete electronic levels, as expected for a spheric quantum dot, it is very surprising that the density dependence of the spectra indicates a gradual filling of the $\omega_{1}$ and $\omega_{4}$ bleaching bands, beginning with the lowest energies. Similar results have been presented in $[6$, 19]. Peyghambarian et al. [19] have considered the case of comparable homogeneous and inhomogeneous broadening and pumping at the lowest absorption line. They explained the shift of the bleaching peak by an increasing bleaching of (homogeneously broadened) transitions that correspond to smaller NCs and have only little overlap with the pump spectrum. Clearly, this can only explain a shift away from the pump maximum, which is not the case in our measurements. Therefore, this band-filling-like behavior is neither consistent with simple homogeneous nor inhomogeneous broadening mechanisms and suggests the existence of a quasicontinuum of electronic levels within the observed bleaching bands.

In addition, the decay dynamics at different probe energies indicate the presence of a fast relaxation process within the bleaching bands that occurs on a time scale of $\leq 200 \mathrm{fs}$, according to the fast-scan data. Similar results in the timeresolved luminescence of polymers have recently been interpreted as a spectral relaxation within an inhomogeneous density of states [40]. However, this effect can be ruled out in the case of semiconductor-doped glass, since the large separation of NCs prevents a strong interaction of NCs with different sizes.

Qualitatively, these results can be interpreted in terms of a strong coupling of the discrete electronic states with a quasi-continuum of vibronic or surface states. It has been shown that a strong electron-phonon coupling exists in semiconductor NCs which can be even stronger than in bulk $\mathrm{CdSe}$ and that coupling with acoustic phonons dominates the optical transitions at room temperature $[5,14]$. In addition, the "single cluster" absorption and emission spectra obtained in $[4,13]$ show pronounced LO-phonon progressions with comparable intensity as the zero-phonon line. This indicates a strong electron-phonon interaction, which means that pure electronic states do not exist, and the material has to be described by a distribution of coupled electron-phonon states [41].

For the case of LO phonons, e.g., the oscillator strength of the optical transition will be distributed in a series of equally spaced phonon-assisted transitions that correspond to the creation of one electron-hole pair plus a certain number of phonons. These will merge into a single absorption band when the linewidths and the inhomogeneous broadening are sufficiently large. Similarly, a coupling of the discrete quantum-dot electronic states with a quasi-continuum of surface states, as proposed in [13], will lead to the formation of states with mixed character.

In each case, the coupling would lead to a certain quasicontinuous density-of-states associated with each discrete electronic level, within which an energy relaxation, e.g., vibronic or electronic relaxation, should be possible. Unfortunately, the experimental data do not allow a determination of the dominant coupling process, even if there exists a single one. Also, an exact theory that takes into account realistic electron-hole wavefunctions and all electron-phonon 
interactions is not yet available and will surely be a major challenge.

\section{Conclusion}

In conclusion, we present experimental data on absorption saturation of CdSe nanocrystals in a glass matrix, after photodarkening by exposure to high-intensity laser pulses. The nanocrystal size is on the order of the bulk exciton Bohr radius, causing a pronounced blue-shift of the lowest optical transition due to carrier confinement. We investigate the excitation density dependence and the dynamics of carrierinduced absorption changes following femtosecond optical excitation. Our data indicate that energy relaxation of the photoexcited carriers occurs with a time constant of $200 \mathrm{fs}$ and recombination occurs on a time scale of a few picoseconds. In contrast to the linear absorption, the nonlinear absorption spectra show several discrete structures, which allow an identification of the lowest optical transitions. The density dependence of the nonlinear absorption provides information on the energy levels that are bleached by pumpinduced carriers. At low pump intensity and long pumpprobe delay times, we observe only two bleaching bands, which show the same density and time dependence. We find that the absorption saturation at room temperature is predominantly related to the occupation of electron rather than hole states; due to a much lower energy splitting of the hole states, photoexcited holes are distributed over a much larger number of levels than the electrons. Therefore, the hole occupation number $f_{\mathrm{h}}$ remains much smaller than the electron occupation number $f_{\mathrm{e}}$. The structures observed in the nonlinear absorption spectra can be assigned to dipole-allowed optical transitons associated with the lowest two electron states in a strong-confinement approximation. In spite of the occurrence of discrete structures, the DTS show a band-filling-like behavior that indicates the presence of quasi-continuous energy bands rather than purely discrete levels. The experimentally observed blue-shift of the bleaching maxima with increasing density can be interpreted as an increasing thermal filling of these bands. The energy dependence of the decay dynamics indicates the existence of a relaxation process within this quasi-continuum, which may be due to a strong coupling of the discrete quantum-dot states to a quasi-continuum of vibronic or surface states.

Acknowledgements. The authors wish to thank G. C. Cho for technical assistance, H. J. Bakker for useful discussions, V. Karavanskii for Raman measurements, and N. Maslov for TEM measurements. V.K. gratefully acknowledges the support from the Alexander-von-Humboldt Stiftung. This work has been supported by the Alfred-Krupp Stiftung.

\section{References}

1. H. Haug (ed.): Optical Nonlinearities and Instabilities in Semiconductors (Academic, Boston 1988)

2. A. I. Ekimov, Al. L. Efros, A. A. Onushchenko: Solid State Commun. 56, $921(1985)$

3. L. Brus: IEEE J. QE 22, 1909 (1986)

4. M. G. Bawendi, W. L. Wilson, L. Rothberg, P. J. Carroll, T. M. Jedju, M. L. Steigerwald, L.E. Brus: Phys. Rev. Lett. 65, 1623 (1990)

5. P. Roussignol, D. Ricard, C. Flytzanis, N. Neuroth: Phys. Rev. Lett. 62, $312(1989)$
6. S. H. Park, R. A. Morgan, Y. Z. Hu, M. Lindberg, S. W. Koch, N. Peyghambarian: J. Opt. Soc. Am. B 7, 2097 (1990)

7. C. Spiegelberg, F. Henneberger, J. Puls: Superlatt. Microstruct. 9, 487 (1991)

8. A. I. Ekimov, F. Hache, M. C. Schanne-Klein, D. Ricard, C. Flytzanis, I. A. Kudryatsev, T. V. Yazeva, A. V. Rodina, Al. L. Efros: J. Opt. Soc. Am. B 10, 100 (1993)

9. S. Gaponenko, U. Woggon, M. Saleh, W. Langbein, A. Uhrig, M. Müller, C. Klingshirn: J. Opt. Soc. Am. B 10, 1947 (1993)

10. D. J. Norris, A. Sacra, C. B. Murray, M. G. Bawendi: Phys. Rev. Lett. 72, 2612 (1994)

11. V. L. Colvin, J. J. Shiang, W. Hoheisel, C. Johnsona, R. W. Schoenlein, D. W. Mittleman, S. J. Rosenthal, C. V. Shank, A. P. Alivisatos: In: Ultrafast Phenomena IX, ed. by P. F. Barbara, W. H. Knox, G.A. Mouron, A. H. Zewail, Springer Ser. Chem. Phys., Vol. 60 (Springer, Berlin, Heidelberg 1994) p. 351

12. R. Cingolani, C. Moro, D. Manno, M. Striccoli, C. DeBlasi, G. C. Righini, M. Ferrara: J. Appl. Phys. 70, 6898 (1991)

13. M. G. Bawendi, P. J. Caroll, W. L. Wilson, L. E. Brus: J. Chem. Phys. 96, $946(1992)$

14. D. M. Mittleman, R. W. Schoenlein, J. J. Shiang, V. L. Colvin, A. P. Alivisatos, C. V. Shank: Phys. Rev. B 49, 14435 (1994)

15. M. Kull, J.-L. Coutaz; J. Opt. Soc. Am. 7, 1463 (1990)

16. U. Woggon, S. Gaponenko, W. Langbein, A. Uhrig, C. Klingshirn: Phys. Rev. B 47, 3684 (1993)

17. Y. V. Vandyshev, V. S. Dneprovskii, V. I. Klimov: Sov. Phys. JETP 74, 144 (1992)

18. V. S. Dneprovskii, V. A. Karavanskii, V. I. Klimov: Phys. Solid State 35, 1297 (1993)

19. N. Peyghambarian, B. Fluegel, D. Hulin, A. Migus, M. Joffre, A. Antonetti, S. W. Koch, M. Lindberg: IEEE J. QE 25, 2516 (1989)

20. R. W. Schoenlein, D. M. Mittleman, J. J. Shiang, A. P. Alivisatos, C. V. Shank: Phys. Rev. Lett. 70, 1014 (1993)

21. P. Roussignol, D. Ricard, J. Lukasik, C. Flytzanis: J. Opt. Soc. Am. B 4, 5 (1987)

22. J. P. Zheng, H. S, Kwok: Appl. Phys. Lett. 54, 1 (1989)

23. P. Maly, F. Trojanek, A. Svoboda: J. Opt. Soc. Am. 10, 1890 (1993)

24. S. Schmitt-Rink, D. A. B. Miller, D. S. Chemla: Phys. Rev. B 35, 8113 (1987)

25. H. Haug, S. W. Koch: Quantum Theory of the Electronic and Optical Properties of Semiconductors (World Scientific, Singapore 1993)

26. L. Banyai, P. Gilliot, Y. Z. Hu, S. W. Koch: Phys. Rev. B 45, 14136 (1992)

27. Y. Z. Hu, S. W. Koch, M. Lindberg, N. Peyghambarian, E. L. Pollock, F. F. Abraham: Phys. Rev. Lett. 64, 1805 (1990)

28. Y. Z. Hu, M. Lindberg, S. W. Koch: Phys. Rev. B 42, 1713 (1990)

29. D. Ricard, M. Ghanassi, M. C. Schanne-Klein: Opt. Commun. 108, 311 (1994)

30. D. B. Tran Thoai, Y. Z. Hu, S. W. Koch: Phys. Rev. B 42, 4137 (1990)

31. K. I. Kang, B. P. McGinnis, Sandalphon, Y. Z. Hu, S. W. Koch, N. Peyghambarian, A. Mysyrowicz, L. C. Liu, S. H. Risbud: Phys. Rev. B 45, 3465 (1992)

32. T. Dekorsy, T. Pfeifer, W. Kütt, H. Kurz: Phys. Rev. B 47, 3842 (1993)

33. V. Klimov, S. Hunsche, H. Kurz: Phys. Rev. B 50, 8110 (1994)

34. F. de Rougemont, R. Frey, P. Roussignol, D. Ricard, C. Flytzanis: Appl. Phys. Lett. 50, 1619 (1987)

35. V. S. Dneprovskii, Al. L. Efros, A. I. Ekimov, V. I. Klimov, I. A. Kudriavtsev, M. G. Novikov: Solid State Commun. 74, 555 (1990)

36. M. Ghanassi, M. C. Schanne-Klein, F. Hache, A. I. Ekimov, D. Ricard, C. Flytzanis: Appl. Phys. Lett. 62, 78 (1992)

37. We used the following effective masses: electrons: $0.11 m_{e}$; heavy holes: $1.0 m_{\mathrm{e}}$; light holes: $0.3 m_{\mathrm{e}}$; split-off holes: $0.48 m_{\mathrm{e}}$

38. This is in contrast with [33], where we adopted the notation of [8]

39. S. Hunsche, H. Heesel, A. Ewertz, H. Kurz, J. H. Collet: Phys. Rev. B 48, 17818 (1993)

40. R. Kersting, U. Lemmer, R. F. Mahrt, K. Leo, H. Kurz, H. Bässler, E. O. Göbel: Phys. Rev. Lett. 70, 3820 (1993)

41. C. B. Duke, G. D. Mahan: Phys. Rev. A 139, 1965 (1965) 